\title{
DESENVOLVIMENTO DE UM CONVERSOR INTERLEAVED BOOST EM HARDWARE IN THE LOOP CONTROLADO POR DSP PARA APLICAÇÕES EM ELETROCOALESCÊNCIA
}

Caio Müller-muller.caio@engenharia.ufjf.br

Universidade Federal do Rio de Janeiro

Rua Horácio Macedo, Bloco H

219471-972 - Rio de Janeiro - RJ

Luís Guilherme Barbosa Rolim - rolim@ufrj.br

Universidade Federal do Rio de Janeiro

Rua Horácio Macedo, Bloco H

219471-972 - Rio de Janeiro - RJ

Aline Damm Falcão Martins - alinedamm@gmail.com

Universidade do Estado do Rio de Janeiro

Rua São Francisco Xavier, $n^{\circ} 524$

20550-900 - Rio de Janeiro - RJ

Resumo: Com o passar dos anos, avanços na área de eletrocoalescência ocorreram e uma melhor definição de Campo Elétrico Crítico apareceu. Para otimizar o processo de eletrocoalescência, a tensão aplicada na fonte deve permanecer o mais próximo possível do campo elétrico crítico e não ultrapassar o campo elétrico de quebra, que é o momento em que o processo perde a otimização, considerado pior do que se não fosse aplicada nenhum campo elétrico. Os sistemas de eletrocoalescedores industriais trabalham com voltagem na faixa de quilovolts. $O$ presente trabalho apresenta uma alternativa para substituir esses eletrocoalescedores pela utilização de conversores de eletrônica de potência. O conversor interleaved boost pode chegar a altas tensões, assim não é necessária uma fonte de tensão elevada, valores menores de tensão na entrada podem ser convertidos em valores ideais de saída escolhendo os componentes e taxa de chaveamento certos. Para controlar esta taxa de chaveamento, a escolha do Digital Signal Processor (DSP) se faz pela sua confiabilidade, preço e velocidade de atuação no controle do conversor para que se mantenha no ponto ótimo. Os resultados simulados por hardware in the loop indicam que mesmo tendo uma sobretensão alta no início, o DSP foi capaz de controlar o sistema.

Palavras-chave: Eletrocoalescência, Conversor Interleaved Boost, DPS.

\section{INTRODUÇÃO}

O primeiro uso da palavra eletrocoalescência foi em uma patente nos Estados Unidos da América por Frederick Gardner Cottrel. Ele propôs utilizar campo elétrico para separação de emulsão de água em óleo (COTTRELL, 1911). Por ser uma área ainda bastante nebulosa, os avanços em eletrocoalescência foram estagnados até a última década quando novas áreas de conhecimentos foram se integrando a este problema e dando novos passos para melhor entendimento do assunto. 
Dentro desse processo, existem 3 pontos importantes de valores de campo elétrico que aceleram ou freiam a separação da emulsão de água/óleo (SOUZA, 2018). Os pontos mencionados são denominados: Início da Coalescência, onde começa o movimento de gotículas de água imersas no óleo; Campo Elétrico Crítico (CEC), onde as gotículas de água se alinham formando um colar e curto-circuitam os eletrodos, se unindo e formando gotículas maiores; Campo Elétrico de Quebra, ou Break-up Eletric Field (BEF), onde as gotículas de água maiores quebram seu invólucro e expelem as gotículas de água menores de seu interior, piorando o processo.

Na literatura, vários autores como (SKODVIN, SJOBLOM, 1996), (SILSET et al., 2010), (WANG et al., 2010) tentam explicar o que é este campo elétrico crítico em função de algumas áreas do conhecimento. BRASIL (2019) em sua tese, compara as informações contidas na corrente elétrica junto com imagens filmadas em uma câmera digital para comprovação de seu trabalho. Estas informações são realçadas por meio da transformada de Wavelet (HSIEH, HUANG, 2000), que graficamente exibe a frequência em função do tempo e da intensidade dessa.

Nesse trabalho, BRASIL (2019) fez testes com distância de eletrodos para 0.1, 0.2 e 0.3 milímetros. Na literatura, encontra-se distâncias de $46 \mathrm{~mm}$ até $203 \mathrm{~mm}$ entre os eletrodos (SUEMAR et al., 2012). Para distâncias de alguns centímetros, a tensão entre esses eletrodos utilizada para que ocorra o processo é de alguns quilovolts.

No ano letivo de 2019, foi proposta na matéria de Aplicações de Microprocessadores em Eletrônica de Potência da pós-graduação da Universidade Federal do Rio de Janeiro, a realização de um projeto onde um microcontrolador irá controlar o chaveamento de um conversor. Este artigo, que resulta do referido projeto, tem como objetivo trabalhar com um conversor interleaved boost em uma tensão inicial de $100 \mathrm{~V}$ elevando-a para uma tensão final de $12.8 \mathrm{kV}$ usando uma distância de uma polegada entre os eletrodos. Há artigos na literatura como o de (Gil et al., 2016) que utilizam esse método para aplicações em sistemas fotovoltaicos. Vários outros artigos apresentam técnicas para controle desse conversor (HENN et al, 2010), (SUDHAKAR BABU, VEERACHARY, 2005) e (HUANG et al., 2003).

O presente trabalho irá simular em um software, um circuito equivalente para o sistema de emulsão e eletrocoalescedor junto com um equipamento chamado Digital Signal Processor (DSP) que irá fazer o controle do disparo dos MOSFETs de um conversor interleaved boost para trabalhar no ponto de Campo Elétrico Crítico desses eletrocoealescedores industriais com tensões na faixa de quilovolts.

A divisão deste artigo é a seguinte: na Seção 2 é apresentada a configuração do sistema; na Seção 3 é apresentado como se encontrou o valor de elevação do conversor; na Seção 4 é apresentado como foi feito o controle; na Seção 5 explica-se como se implementou no DSP a lógica de controle; por fim, na Seção 6 são apresentadas as conclusões do trabalho.

\section{CONFIGURAÇÕES DO SISTEMA}

Um conversor interleaved boost de $1 \mathrm{kVA}$ é apresentado na Figura 1. Os valores de indutor e capacitor encontrados na simulação de malha aberta que apresentaram uma resposta rápida, suave e satisfatória, apesar da sobretensão inicial, foi de $1 \mathrm{mH}$ para os indutores e $2.2 \mathrm{uF}$ para os capacitores.

A escolha de um DSP é justificada pelo rápido tempo de resposta no controle necessário para diminuir as perdas e aumentar a confiabilidade do sistema de operar no ponto de máximo rendimento. 
Figura 1- Representação do Sistema.

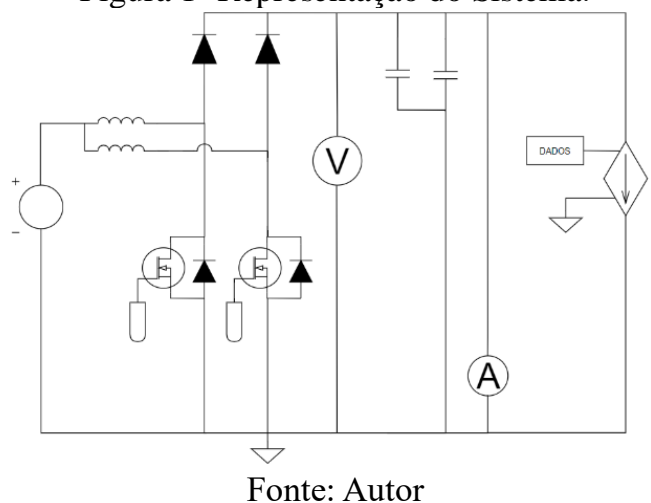

Na literatura, pelo trabalho de (MOHAMMADI et al., 2019), a carga do conversor é apresentada como um conjunto de resistências em paralelo com um capacitor simbolizando o fluxo de óleo com diferentes percentuais de água e os eletrodos. Uma vez que começa a extração de petróleo em um poço offshore, uma porcentagem de água também é drenada junto ao óleo. Quanto maior a idade do poço, maior também é a quantidade de água drenada junto com o óleo. Todavia, a partir de medições feitas em laboratório, optou-se por definir a carga como sendo uma tabela de pontos onde a corrente varia em certos valores de tensão, sendo a média entre esses pontos obtidos experimentalmente já mencionados anteriormente.

As Figuras 2 (a) e 2 (b) ilustram um dos resultados coletados de uma emulsão de óleo Exxol d80 com 10\% de água deionizada, proporção massa-massa em formas de gráficos de tensão (V/mm) por Amplitude (A), e estes após serem tratados por um filtro butterworth de quarta ordem com largura de banda de $20 \mathrm{~Hz}$ e frequência de ripple de $0.005 \mathrm{~Hz}$..

Figura 2 - Resposta da corrente.

(a)

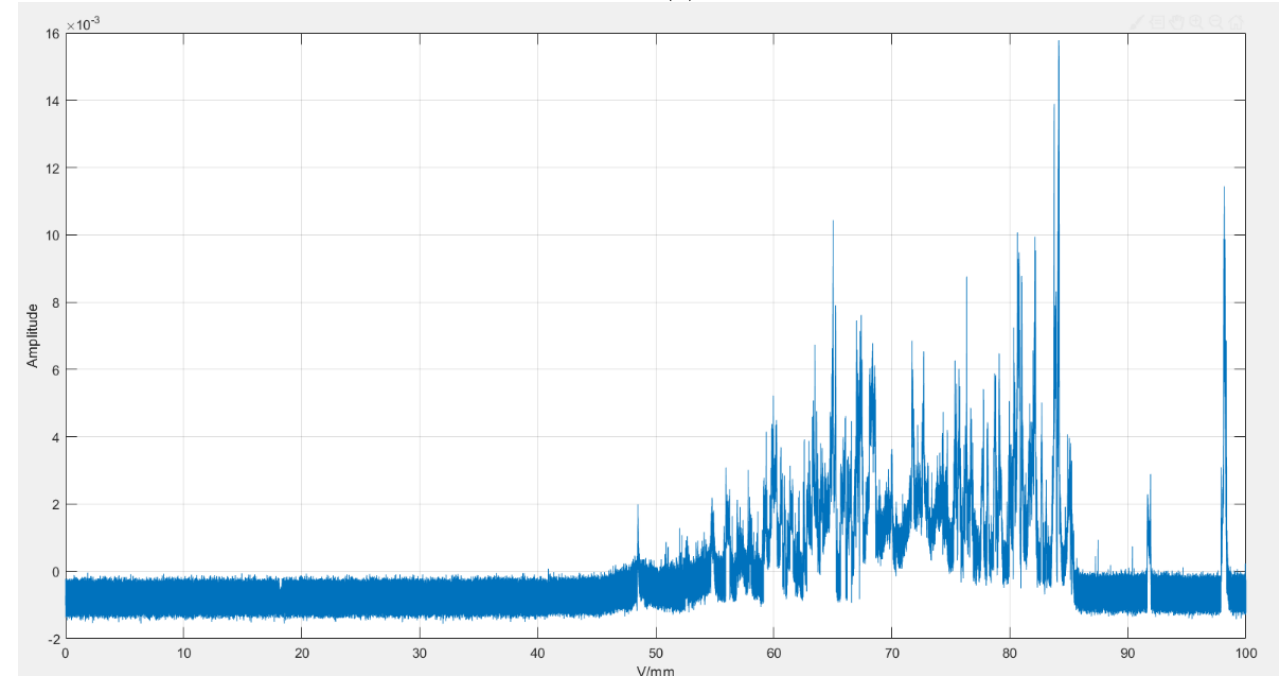


(b)

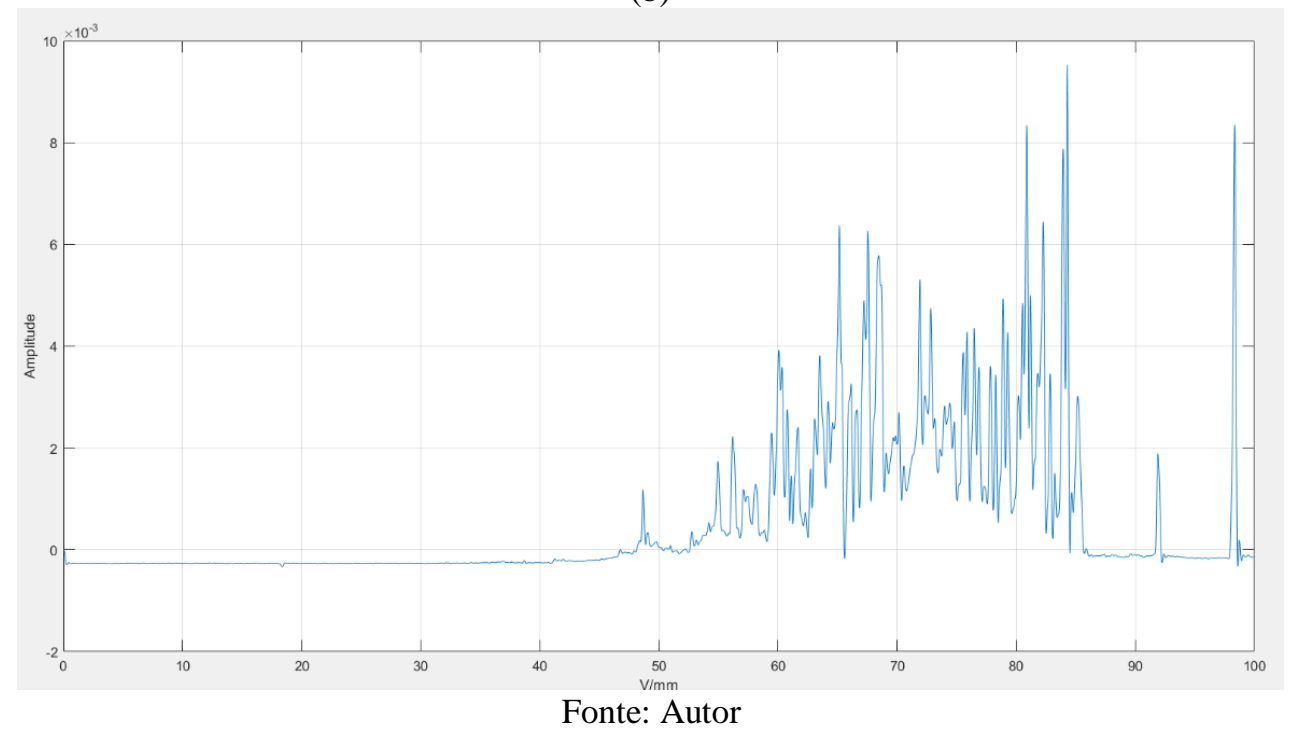

\section{CÁlCULO DO CONVERSOR}

Para valores de distância de eletrodos de $0.1,0.2$ e 0.3 milímetros, a forma de onda da corrente se mantém parecida na razão dos valores de tensão por milímetro. Na literatura também se encontram valores maiores de distância entre eletrodos e o mesmo aspecto da forma de onda da corrente. Assim, considerando uma linearidade no fenômeno, para distâncias na casa dos centímetros, os valores de tensão serão na casa dos quilovolts.

Em seu livro, (MOHAN et al., 2003) apresenta a fórmula da razão entre as tensões de saída e entrada para se obter a razão cíclica utilizada no chaveamento do transistor em um conversor boost. Eis a equação 1 em questão:

$$
\frac{V_{o}}{V_{\text {in }}}=\frac{1}{1-D}
$$

Substituindo-se os valores de entrada e saída por $100 \mathrm{~V}$ e $12.8 \mathrm{kV}$, respectivamente, obtémse uma razão cíclica de 0.992 .

\section{CÁlCULO DO CONTROLE}

A verificação de funcionamento do circuito é feita por um sistema de controle em malha aberta. A Figura 3 ilustra o resultado da tensão e da corrente respectivamente quando não há um controle em malha fechada. Nota-se que o regime permanente ocorre em 1.2 segundo de simulação. 
Figura 3 - Resultado em malha aberta

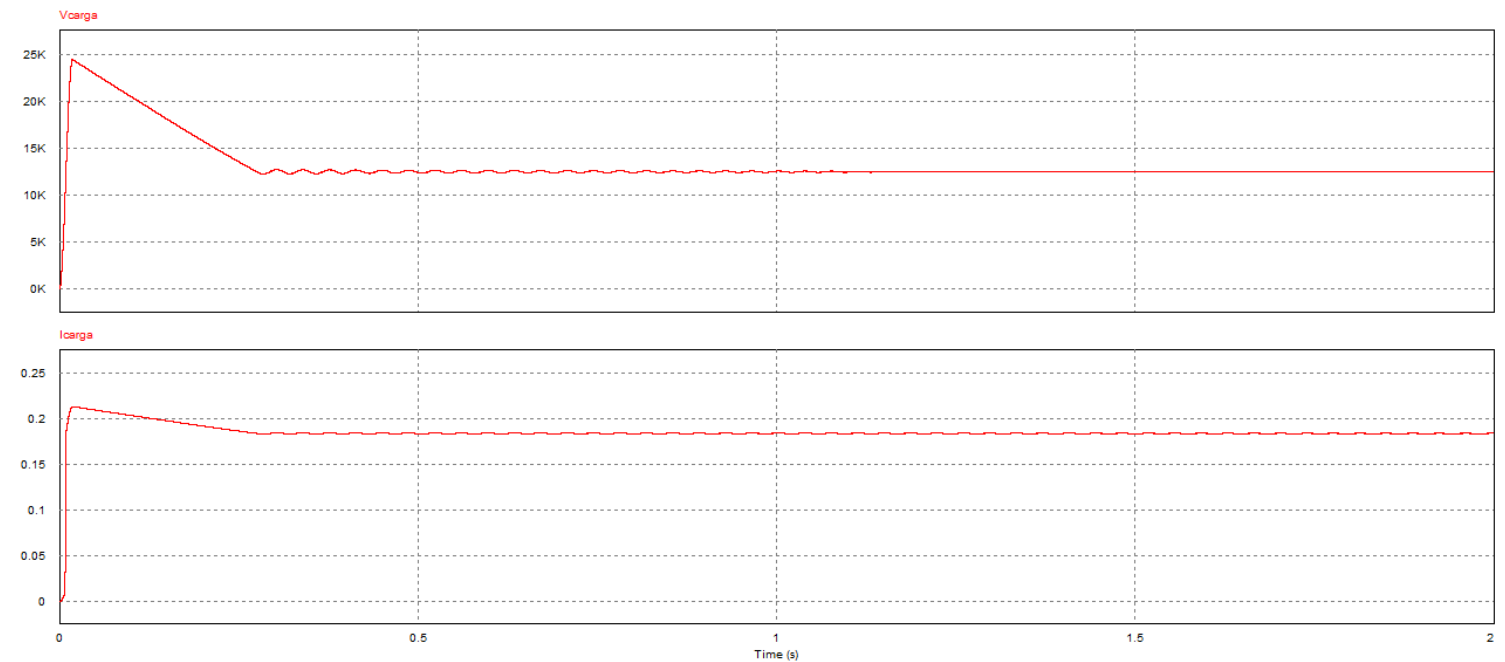

Fonte: Autor

Como é possível ver na Figura 2, há um salto abrupto na corrente em um determinado valor na tensão. Este momento é chamado de Break-Up Eletric Field. O BEF é o ponto onde a eficiência do processo passa de máxima para mínima, podendo fazer, em vários casos, demorar mais tempo para separar a emulsão se caso não acontecesse interferência externa.

O método de controle utilizado para se manter próximo ao ponto de máxima eficiência foi um controle tipo Bang-Bang (BRYSON, HO, 1969). O controlador registra os valores de tensões e, quando há uma variação de corrente abrupta, um novo valor de razão cíclica é calculado para encontrar o ponto de máxima eficiência com uma tolerância de $2.5 \%$ entre o valor de tensão do BEF e a tensão para a razão cíclica.

A Figura 4 apresenta o resultado do controle de malha fechada.

Figura 4 - Resultado em malha fechada

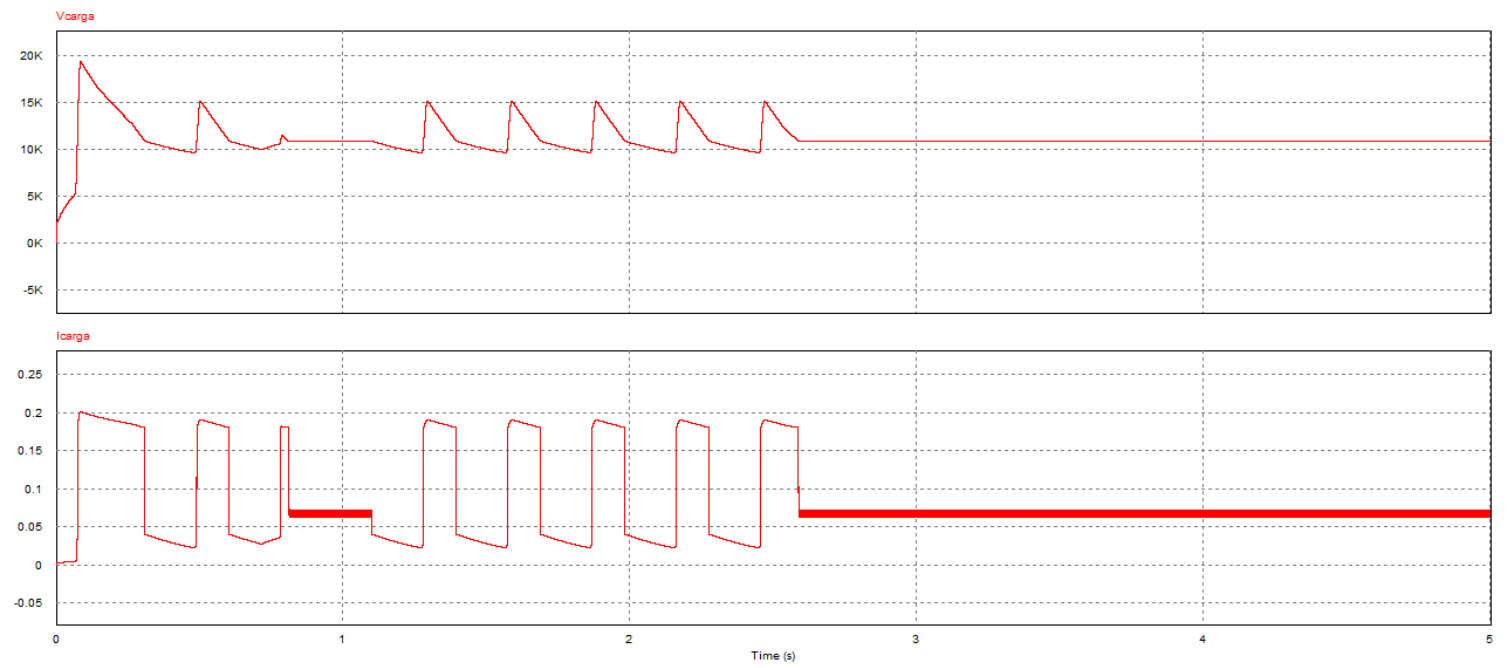

Fonte: Autor

O resultado do controle em malha fechada consegue se estabilizar após um período de tempo mantendo a corrente em um nível aceitável e não permitindo a explosão das gotículas de 
água. Nota-se também a espessura da faixa vermelha de corrente na Figura 4, que representa um ripple de alta frequência e indica que o controle está atuando.

Este resultado é o esperado, pois o novo fluxo que passar pelo eletrocoalescedor, já estará ajustado para próximo do CEC, separando com mais eficiência a emulsão de óleo e água.

\section{IMPLEMENTAÇÃO EM DSP}

O DSP escolhido para controle foi o TMS320F280049 da Texas Instruments. Sua memória de $256 \mathrm{~Kb}$ e seu núcleo com coprocessador de ponto flutuante foram suficientes para o controle em questão.

A Texas Instruments possui um ambiente de desenvolvimento próprio dos seus microcontroladores, o Code Composer Studios (CCS). O código então foi desenvolvido baseado em uma biblioteca exemplo dentro do CCS. Além disso, a ponte entre o CCS e o PSIM é feita por meio da criação de uma DLL pelo Microsoft Visual Studio. A Figura 5 exemplifica a interação entre os ambientes.

Figura 5 - Interação entre os ambientes

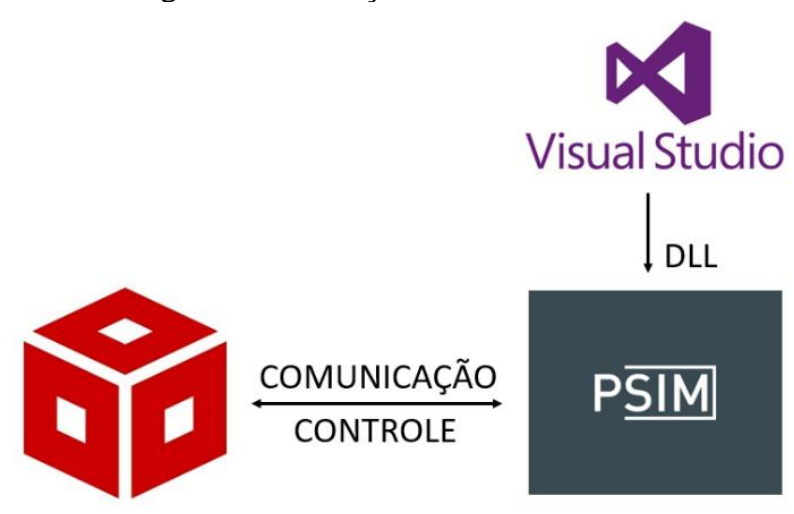

Fonte: Autor

Ao fazer as simulações, acontecia um erro entre processamento de dados e a resposta do microcontrolador. Para solucionar o problema, um bloco de Zero Order Hold foi acoplado ao sistema para converter o sinal digital para analógico e, com isso, os resultados foram satisfatórios.

\section{CONCLUSÃo}

Este trabalhou abordou um novo ponto de vista de uma área não muito conhecida por se tratar de um tema multidisciplinar que integra principalmente as áreas de química, elétrica, eletrônica e processamento de sinais. Muitos autores buscam entender como o processo funciona, este trabalho visa controlar o sistema.

Apesar do fenômeno de separação de emulsão por eletrocoalescência não ter uma fórmula explícita do comportamento das variáveis que influenciam no processo, ainda é possível encontrar e manter o ponto de máxima eficiência.

A estratégia proposta permite a reprodução do experimento ao acoplar a confecção de uma placa de circuito impressa com os componentes do circuito e realizar testes experimentais em bancadas e comparar com os resultados obtidos na simulação. 
O presente trabalho foi concluído com êxito, mostrando que é possível controlar a corrente por meio de técnicas de controle clássicas com auxílio de equipamentos externos.

\section{Agradecimentos}

O presente trabalho foi realizado com apoio da Coordenação de Aperfeiçoamento de Pessoal de Nível Superior - Brasil (CAPES) - Código de Financiamento 001. E à Universidade Federal do Rio de Janeiro.

\section{REFERÊNCIAS}

BRASIL, Thiago Americano. Análise microscópica e avaliação quantitativa do processo de desestabilização de emulsões água-em-óleo com aplicação de campo elétrico. 2019. 132 f. Tese (Doutorado) - Programa de Pós-Graduação em Engenharia Elétrica - COPPE/UFRJ, 2019.

BRYSON, A. E. JR.; HO, Y. Singular Solutions of Optimization and Control Problems. Applied Optimal Control. Waltham: Blaisdell. pp. 246-270, 1969.

COTTRELL, Frederick Gardner. Process for separating and collecting particles of one liquidsuspended in another liquid. US Patent 987.114, 1911.

GIL, G. M. V.; CATATA, E. O. H.; CCARITA, J. C. C.; CARDOSO, J. G.; FILHO, A. J. S.; AZCUE-PUMA, J. L. Digital controller design for interleaved boost converter in photovoltaic system. 12th IEEE International Conference on Industrial Applications - INDUSCON 2016, pp. 1-8, 2017.

HENN, G. A. L.; SILVA, R. N. A. L.; PRAÇA, P. P.; BARRETO, L. H. S. C.; DEMERCIL, D. S. Interleaved-boost converter with high voltage gain. IEEE Transactions. Power Electronics, vol. 25, no. 11, pp. 2753-2761, 2010.

HUANG, X.; NERGAARD, T.; LAI, J. S.; XU, X.; ZHU, L. A DSP based controller for highpower interleaved boost converters. IEEE Application on Power Electronics Conference Exposition - APEC, vol. 1, no. C, pp. 327-333, 2003.

HSIEH, C. T.; HUANG, S. J. Computation of Continuous Wavelet Transform via A New Wavelet Function for Visualization of Power System Disturbances. IEEE Transactions, vol. 00, no. c, pp. 951-955, 2000.

MOHAMMADI, F.; MOHAMMADI, M.; NONAHAL, B. A comprehensive electrical model for the electrostatic desalting process of crude oil. Petroleum and Coal, vol .61, pp. 738-748, 2019.

MOHAN, Ned; UNDELAND, Tore M.; ROBBINS, William P.. Power electronics: converters, applications, and design. Hoboken, $3^{\text {a }}$ edicção, NJ: J. Wiley, 2003.

SILSET, A.; HANNISDAL, A.; HEMMINGSEN, P. V.; SJÖBLOM, J. Emulsions of Heavy Crude Oils. II. Viscous Responses and Their Influence on Emulsion Stability Measurements. J. Dispers. Sci. Technol., vol. 31, no. September 2013, pp. 1432-1445, 2010. 
SKODVIN, T.; SJOBLOM, J. Dielectric spectroscopy on W/O emulsions under influence of shear forces. Colloid Polym. Sci., vol. 274, no. 8, pp. 754-762, 1996.

SOUZA, Troner Assenheimer. Avaliação da estabilidade de emulsões sob campo elétrico utilizando microscopia e planta piloto. 2018. 146 f. Tese (Doutorado) - Programa de PósGraduação em Engenharia de Processos Químicos e Bioquímicos - COPPE/UFRJ, 2018.

SUDHAKAR BABU, C.; VEERACHARY, M. Predictive controller for interleaved boost converter. IEEE International Symposium on Industrial Electronics, vol. II, no. i, pp. 577$582,2005$.

SUEMAR, P.; FONSECA, E. F.; COUTINHO, R. C.; MACHADO, F.; FONTES, R.; FERREIRA, L. C.; LIMA, E. L.; MELO, P. A.; PINTO, J. C.; NELE, M. Quantitative Evaluation of the Efficiency of Water-in-Crude-Oil Emulsion Dehydration by Electrocoalescence in Pilot-Plant and Full-Scale Units. Industrial \& Engineering Chemistry Research. vol. 51. pp. 13423-13437, 2012.

WANG, X.; BRANDVIK, A.; ALVARADO, V. Probing interfacial water-in-crude oil emulsion stability controls using electrorheology. Energy and Fuels, vol. 24, no. 12, pp. 6359$6365,2010$.

\title{
DEVELOPMENT OF AN INTERLEAVED BOOST CONVERTER IN DSP CONTROLLED HARDWARE IN THE LOOP FOR ELECTRALCOALESCENCE APPLICATIONS
}

\begin{abstract}
Over the years, advances in the area of electrocoalescence have occurred and a better definition of Critical Electric Field has appeared. To optimize the electrocoalescence process, the voltage applied to the source must remain as close as possible to the critical electric field and not exceed the break up electric field, which is the moment when the process loses its optimization, considered worse than if there were no electrical field applied. Industrial electrocoalescent systems work with voltage in the kilovolt range. The present work presents an alternative to replace these electrocoalescers with the use of power electronics converters. The interleaved boost converter can reach high voltages, so there is no need for a high voltage source, lower voltage values at the input can be converted into ideal output values by choosing the right components and switching rate. To control this switching rate, the choice of the Digital Signal Processor (DSP) is made due to its reliability, price and speed of action in the control of the converter so that it remains at the optimum point. The results simulated by hardware in the loop indicate that despite having a high overvoltage at the beginning, the DSP was able to control the system.
\end{abstract}

Keywords: Eletrocoalescence, Interleaved Boost Converter, DSP. 